\title{
Patient willingness for repeat screening and preference for CT colonography and optical colonoscopy in ACRIN 6664: the National CT Colonography trial
}

\author{
This article was published in the following Dove Press journal: \\ Patient Preference and Adherence \\ 23 July 2015 \\ Number of times this article has been viewed
}

\section{Ilana F Gareen ${ }^{1,2}$ \\ Bettina Siewert ${ }^{3}$ \\ David J Vanness ${ }^{4}$ \\ Benjamin Herman ${ }^{2}$ \\ CD Johnson ${ }^{5}$ \\ Constantine Gatsonis 2,6}

'Department of Epidemiology, Brown University School of Public Health, Providence, RI, USA; ${ }^{2}$ Center for Statistical Sciences, Brown University School of Public Health, Providence, RI, USA; ${ }^{3}$ Department of Radiology, Beth Israel Deaconess Medical Center Boston, MA, USA; 'Population Health Sciences, University of Wisconsin, Madison, WI, USA; ${ }^{5}$ Mayo Clinic, Scottsdale, AZ, USA; ${ }^{6}$ Department of Biostatistics, Brown University School of Public Health, Providence, RI, USA
Correspondence: llana F Gareen Center for Statistical Sciences, Brown University School of Public Health, Box G-SI2 I-7, Providence, RI 02912, USA

$\mathrm{Tel}+\mid$ 40I 863 I758

Fax + 4018639182

Email igareen@stat.brown.edu
Background: Current American Cancer Society recommendations for colon cancer screening include optical colonoscopy every 10 years or computed tomography colonography (CTC) every 5 years. Bowel preparation (BP) is currently required for both screening modalities.

Purpose: To compare ACRIN 6664: the National CT Colonography Trial (NCTCT) participant experiences with CTC and optical colonoscopy (OC), procedure preference, and willingness to return for each procedure.

Materials and methods: Participants from fifteen NCTCT sites, who underwent CTC followed by $\mathrm{OC}$ under sedation, were invited to complete questionnaires 2 weeks postexam, asking about procedure preference, physical discomfort, and embarrassment experienced and whether that discomfort and embarrassment was better or worse than expected during BP, CTC, and OC, as well as willingness to return for repeat $\mathrm{CTC}$ and $\mathrm{OC}$ at different time intervals.

Results: A total of 2,310 of 2,600 patients $(89 \%)$ returned their questionnaires. Of patients reporting a preference, $1,058(46.6 \%)$ preferred CTC, 569 (25.0\%) preferred OC, and 626 (27.6\%) reported no preference. Participant-reported discomfort worse than expected differed significantly between CTC (32.9\%) and OC (5.0\%) $(P<0.001)$. About 79.3\% were willing to be screened again with CTC in 5 years, and $96.6 \%$ with OC in 10 years. Discomfort and embarrassment worse than expected with OC were associated with increased intention to adhere with CTC in the future. Conversely, embarrassment experienced during CTC and discomfort worse than expected on CTC were associated with increased intention to adhere with OC in the future.

Conclusion: While a larger proportion of participants indicated that they preferred CTC to OC, willingness to undergo repeat CTC compared to OC was limited by unanticipated exam discomfort and embarrassment and CTC's shorter screening interval.

Keywords: CT colonography, colonoscopy, bowel preparation, adherence, patient preference

\section{Introduction}

Colorectal cancer is a major cause of morbidity and mortality in the United States, with 143,460 new cases and 51,690 deaths attributed to colorectal cancer in 2012. ${ }^{1}$ The US Preventive Services Task Force currently recommends that adults aged 50-75 at low-risk for colon cancer receive regular screening according to one of three regimens: 1) annual screening with high-sensitivity fecal occult blood testing (FOBT); 2) sigmoidoscopy every 5 years, with high-sensitivity FOBT every 3 years; or 3) screening colonoscopy every 10 years., ${ }^{2,3}$ Colorectal cancer mortality reduction has been demonstrated for FOBT, sigmoidoscopy, and colonoscopy with 
polyp removal..$^{4-9}$ Despite these recommendations, many of those at highest risk of colon cancer fail to get screened, ${ }^{10-12}$ and many of those who do get screened fail to adhere to the recommended screening schedule. ${ }^{12}$ In a recently published Canadian survey, only $23.6 \%$ of the study population had received any colon cancer screening, and only $17.6 \%$ had received screening according to the currently recommended schedule. ${ }^{3,11,13}$ Optical colonoscopy (OC) visualizes the entire colon and offers excellent sensitivity, specificity, the opportunity to obtain biopsy samples at the time of screening, a long screening interval (10 years), and a high degree of accuracy. ${ }^{3} \mathrm{OC}$ is regarded as the gold standard for colon cancer screening. Despite these advantages, OC is invasive and carries some risk of bowel perforation ${ }^{13}$ and anesthesiaassociated complications. ${ }^{14}$

Computed tomography colonography (CTC) screens for colon cancer by visualizing the entire colon while avoiding an invasive procedure and sedation, minimizing risk of bowel perforation, and shortening patient time away from regular activities. ${ }^{15}$ Like OC, CTC currently requires a cathartic bowel preparation (BP), although current research is underway to eliminate the need for BP. ${ }^{16-19}$ Current CTC protocols require the colon to be inflated with air through the rectum, a procedure that many patients find uncomfortable and embarrassing. In addition, CTC has a shorter recommended screening interval (5 years) than OC, although there is evidence that this shorter screening interval may not raise a barrier to repeat screening with CTC as opposed to OC. ${ }^{20}$

Patient expectations of the discomfort and embarrassment associated with CTC, as opposed to OC, may not be entirely accurate. In a recent study in which participants were randomly assigned to be offered screening with CTC or OC, the expected burden of screening was higher for those invited for OC than for those invited for CTC, and a significantly higher proportion of those invited for CTC attended the assigned screening examination than did those invited for OC. However, after screening, the embarrassment, pain, and perceived burden of the entire screening procedure were higher for CTC participants than for OC participants. Despite these perceived differences, the CTC and OC group were comparable with respect to their expressed intention to attend the next screening round. ${ }^{21}$

The purpose of this study was to examine the experience of average-risk participants undergoing both CTC and OC as part of a screening study and to assess the effect of embarrassment, discomfort, and other factors on their willingness to return for repeat screening with each procedure.

\section{Materials and methods}

Participants were drawn from the population enrolled in ACRIN 6664: the National CT Colonography Trial (NCTCT). The NCTCT was led by the American College of Radiology Imaging Network (ACRIN), which is now a part of the Eastern Cooperative Oncology Group (ECOG)-ACRIN Cancer Research Group. The NCTCT has been described in detail elsewhere. ${ }^{22}$ Participants underwent a single BP and were scheduled to receive CTC, followed by sedation and OC. Asymptomatic patients, 50 years of age or older, who were scheduled to undergo OC were recruited from February 2005 to December 2006. All patients provided informed consent. The template consent advised participants that:

For the CT colonography, you will be taken into the CT room and asked to lie on your side. An enema tip will be placed in your rectum and your colon will be slowly inflated with air (carbon dioxide) until you feel full.

Each patient was required to have a fully prepared colon. Colonic insufflation was obtained with an automated carbon dioxide insufflator (PROTOC02L, E-Z-EM Inc., Lake Success, NY, USA). Manual insufflation with room air was used if adequate colon distention could not be obtained with the mechanical insufflator. One milligram of subcutaneous glucagon was administered $7-15$ minutes before the examination unless contraindicated or declined by the study participant. ${ }^{22}$ Further details are provided in the study protocol. ${ }^{23}$

For this substudy, we mailed a participant questionnaire approximately 2 weeks after CTC and OC were completed. ${ }^{24}$ We chose this time interval with the hope that it was recent enough for participants to recall their experience, but distant enough that patients would have been notified of their results prior to completing questionnaires. For 13 of 15 study sites, questionnaires were mailed from the ACRIN Outcomes and Economics Assessment Unit located at Brown University. At two sites, due to institutional review board privacy concerns, study site research associates mailed the questionnaires directly to the participants.

The questionnaire included questions asking participants to evaluate the $\mathrm{BP}$, the $\mathrm{CTC}$, and the $\mathrm{OC}$ and rate the actual discomfort and embarrassment that they experienced (none, mild, moderate, severe, worst ever), as well as to compare their actual experience to their expectations of discomfort and embarrassment (much better, somewhat better, about the same, somewhat worse, much worse). Participants were also asked how often they would be willing to return for a repeat screening examination with each test (CTC, OC), whether the minimum acceptable interval between tests would be altered if a BP were 
unnecessary, which procedure they preferred, whether they had a preference for the sex of the examiner, and the time and expenses incurred to attend the screening session.

Permission to conduct this study was obtained from the Brown University and each site's institutional review boards (Supplementary material).

\section{Statistical analysis}

Data were analyzed by the ACRIN Biostatistics Center, located at the Brown University Center for Statistical Sciences (Providence, RI, USA). Descriptive data were tabulated and simple binomial 95\% confidence limits were estimated. We used McNemar's test, which accounted for the paired nature of the data in which each patient was screened with both modalities, to compare CTC with OC with respect to dichotomized participant responses on willingness to undergo repeat screening with differing screen intervals, actual discomfort and embarrassment experienced, actual as compared with expected discomfort and embarrassment experienced, and patient preference for the sex of the examiner at future screenings.

We used polytomous logistic regression to examine the relationship between a participant's willingness to return for screening within the recommended interval and participantreported actual and actual as opposed to expected discomfort and embarrassment related to the BP, CTC, and colonoscopy, time and travel involved in obtaining the screening examination, and whether removing the need for BP would alter willingness to return. We did not adjust for the results of the two examinations as the CTC was performed as an experimental procedure, and results were reported to participants at the site's discretion.

In these models, the response was a four-category variable indicating willingness to return for screening with CTC only, with OC only, with both modalities, or with neither modality. We defined planned adherence as a patient reporting a willingness to be rescreened within the recommended interval for each test ( 5 years for CTC and 10 years for OC); respondents were categorized as planning to adhere to CTC only, OC only, both tests, or neither test. Reported odds ratios (ORs) should be interpreted as the odds of a patient planning to be adherent with only CTC, with only OC, or with neither test, relative to the odds of planning to be adherent with both tests. The independent variables included all covariates described above plus participant sociodemographic information.

We used the following process to arrive at our final model. If the conservative $P$-value was less than 0.2 , that independent variable was retained as a candidate for the final model. The final model was obtained using the filtered list of independent variables as well as age and sex in a backwards logistic regression fitting. We included whether the participant had had a biopsy during the colonoscopy as an a priori confounder. Calculations were performed using the statistical software SAS 9.1 (SAS Institute, Cary, NC, USA).

\section{Results}

Characteristics of the study population are reported in Table 1. Of 2,600 patients screened in the NCTCT, 2,310 (89\%: 1,224 women, 1,086 men) completed the patient questionnaire. Mean age was 58.4 years (range: $50-86$ years). The participant population was $84.8 \%$ Caucasian, $11.1 \%$ African American, and $4.1 \%$ other/unknown. Those who responded were more likely to be older, female, and white. Not all participants provided information for all questions, and we chose to use all available data in the tables.

About 46.6\% (95\% confidence interval [CI]: 44.5\%$48.7 \%$ ) responded that they preferred CTC, $25.0 \%(95 \%$ CI: $23.3 \%-26.9 \%$ ) responded that they preferred OC, $27.6 \%$ (95\% CI: $25.8 \%-29.5 \%$ ) responded that they had no preference, and less than $1 \%(95 \% \mathrm{CI}: 0.5 \%-1.3 \%)$ responded that they would not return for either test. These responses, however, were inconsistent with participant-reported planned adherence for each test (Table 2). Despite $46.6 \%$ of participants reporting that they preferred CTC, only $79.3 \%$ of the

Table I Comparison of participants with information available on patient preference compared with those for the entire trial

\begin{tabular}{llll}
\hline Variable & $\begin{array}{l}\text { Analysis set } \\
(\mathbf{N}=\mathbf{2 , 3 1 0 )}\end{array}$ & $\begin{array}{l}\text { Failed to return } \\
\text { questionnaire (N=290) }\end{array}$ & $\begin{array}{l}\text { Accrued into main } \\
\text { study (N=2,600) }\end{array}$ \\
\hline $\begin{array}{l}\text { Age, mean (range) } \\
\text { Sex, } \mathrm{n}(\%)\end{array}$ & $58.39(50-86)$ & $57.34(50-80)$ & $58.27(50-86)$ \\
$\quad \begin{array}{l}\text { Female } \\
\text { Male }\end{array}$ & $1,224(52.9 \%)$ & $147(50.6 \%)$ & $1,371(52.7 \%)$ \\
Race, $\mathrm{n}(\%)$ & $1,086(47.0 \%)$ & $143(49.3 \%)$ & $1,229(47.2 \%)$ \\
$\quad$ White & $1,961(84.8 \%)$ & $183(63.1 \%)$ & $2,144(82.4 \%)$ \\
$\quad$ Black or African American & $257(11.1 \%)$ & $86(29.6 \%)$ & $343(13.1 \%)$ \\
$\quad$ Other & $97(4.1 \%)$ & $21(7.2 \%)$ & $118(4.5 \%)$ \\
\hline
\end{tabular}


Table 2 Willingness of participants to undergo repeat screening with computed tomography colonography (CTC) and optical colonoscopy (OC)

\begin{tabular}{|c|c|c|c|c|c|c|c|c|c|c|}
\hline \multirow[t]{3}{*}{ Time interval } & \multicolumn{5}{|c|}{ With bowel preparation } & \multicolumn{5}{|c|}{ Without bowel preparation } \\
\hline & \multicolumn{2}{|l|}{ CTC } & \multicolumn{2}{|l|}{ OC } & \multirow[t]{2}{*}{$P$-value } & \multicolumn{2}{|l|}{ СТC } & \multicolumn{2}{|l|}{ OC } & \multirow[t]{2}{*}{$P$-value } \\
\hline & $\mathbf{N}$ & $\%$ & $\mathbf{N}$ & $\%$ & & $\mathbf{N}$ & $\%$ & $\mathbf{N}$ & $\%$ & \\
\hline Every year & 368 & $16.1 \%$ & 328 & $14.4 \%$ & 0.001 & 1,133 & $49.8 \%$ & 864 & $38.0 \%$ & $<0.001$ \\
\hline Every 3 years & $\mathrm{I}, 026$ & $45.0 \%$ & 974 & $42.7 \%$ & 0.003 & 1,694 & $74.5 \%$ & $\mathrm{I}, 556$ & $68.4 \%$ & $<0.001$ \\
\hline Every 5 years & 1,809 & $79.3 \%$ & I,825 & $80.0 \%$ & 0.368 & 2,055 & $\mathbf{9 0 . 4 \%}$ & 2,057 & $90.5 \%$ & 0.946 \\
\hline Every 10 years & 2,105 & $92.2 \%$ & 2,204 & $96.6 \%$ & $<0.001$ & 2,154 & $94.7 \%$ & 2,208 & $97.1 \%$ & $<0.001$ \\
\hline Never & 143 & $6.3 \%$ & 57 & $2.5 \%$ & $<0.001$ & 105 & $4.6 \%$ & 56 & $2.5 \%$ & $<0.001$ \\
\hline
\end{tabular}

Notes: Numbers in bold represent a comparison of intended adherence to recommended screening intervals ( 5 yearly CTC vs I0 yearly OC). $P<0.00 \mathrm{I}$ for both comparisons: with and without bowel preparation.

participants indicated that they were willing to be screened again with CTC in 5 years (the recommended interval for CTC), as opposed to $96.6 \%$ who indicated that they were willing to be screened again with $\mathrm{OC}$ in 10 years (the recommended interval for $\mathrm{OC})(P<0.001)$. If the screening interval for CTC was extended to 10 years, $92.2 \%$ of the participants were willing to return for CTC screening as opposed to $96.6 \%$ for $\mathrm{OC}$ screening $(P<0.001)$. If $\mathrm{BP}$ were unnecessary, $90.4 \%$ were willing to be rescreened with CTC in 5 years and $97.1 \%$ were willing to be rescreened with OC in 10 years $(P<0.001)$.

Discomfort and embarrassment associated with BP and each test are shown in Table 3. Participants were more likely to report experiencing more discomfort with CTC than with OC $(81.3 \%$ vs $27.8 \%, P<0.001)$ as well as more embarrassment with CTC than with OC ( $42.5 \%$ vs $26.0 \%, P<0.001)$. In addition, significantly more participants reported that the actual discomfort experienced was worse than expected for CTC than for OC $(32.9 \%$ vs $5.0 \%, P<0.001)$ as was the embarrassment experienced with CTC as opposed to OC ( $8.5 \%$ vs $1.6 \%, P<0.001)$.

Women were more likely to express a preference for the sex of the examiner when having the CTC examination than when undergoing OC. Although both men and women were more likely to express a preference for a female examiner for the CTC than for the OC examination, this preference was substantially more prevalent among women than among men (Table 4).

The results of a single polytomous logistic regression model exploring the factors associated with planned adherence with recommended screening guidelines with both modalities, CTC only, OC only, or neither are shown in Table 5. A total of 2,086 of 2,310 participants had complete data available for this analysis. ORs greater than one in the column titled "Plan to adhere with neither" indicate factors associated with a general unwillingness to be rescreened within the recommended interval for either test relative to the odds of planning to be adherent with both tests. A greater unwillingness to be rescreened with either test relative to being willing to be rescreened with both tests was associated with older age (OR [10-year increase, starting at age 50] $=1.78 ; 95 \%$ CI: 1.11-2.85), female sex $(\mathrm{OR}=2.46$; 95\% CI: 1.06-5.72), worse than expected discomfort from BP (OR=9.50; 95\% CI: 4.41-20.48), discomfort on CTC (OR=3.78; 95\% CI: 1.01-14.17), worse than expected discomfort on OC (OR=3.54; 95\% CI: 1.38-9.05), and worse than expected embarrassment on $\mathrm{OC}(\mathrm{OR}=18.50 ; 95 \% \mathrm{CI}$ : 5.30-60.48).

Relative intentions to return for screening with each specific modality (OC and CTC) within the recommended time intervals relative to the odds of planning to be adherent with both tests is shown in Table 5, columns titled "Plan to

Table 3 Discomfort experienced, comparison of actual discomfort compared with expectations, embarrassment experienced, and comparison of actual embarrassment compared with expectations for bowel preparation (BP), CT colonography (CTC), and optical colonoscopy (OC), and comparison of CTC and OC with respect to each parameter

\begin{tabular}{|c|c|c|c|c|c|c|c|c|}
\hline & \multicolumn{2}{|l|}{ BP } & \multicolumn{2}{|l|}{ CTC } & \multicolumn{2}{|l|}{ OC } & \multirow{2}{*}{$\frac{\text { CTC vs OC }}{P \text {-value* }}$} & \multirow{2}{*}{$\frac{\text { Respondent }}{\mathbf{N}^{\mathrm{a}}}$} \\
\hline & $\mathbf{N}$ & $\%$ & $\mathbf{N}$ & $\%$ & $\mathbf{N}$ & $\%$ & & \\
\hline Any discomfort experienced & 1,886 & $83.4 \%$ & $\mathrm{I}, 838$ & $81.3 \%$ & 628 & $27.8 \%$ & $<0.001$ & 2,261 \\
\hline Discomfort worse than expected & 426 & $19.0 \%$ & 740 & $32.9 \%$ & 112 & $5.0 \%$ & $<0.001$ & 2,247 \\
\hline Any embarrassment experienced & 554 & $24.3 \%$ & 971 & $42.5 \%$ & 593 & $26.0 \%$ & $<0.001$ & 2,284 \\
\hline Embarrassment worse than expected & 104 & $4.8 \%$ & 185 & $8.5 \%$ & 35 & $1.6 \%$ & $<0.001$ & 2,185 \\
\hline
\end{tabular}

Notes: *Exact $P$-values from McNemar's test. alncludes only cases with responses to all three modalities. 
Table 4 Patient's preference for the sex of the examiner by screening modality: computed tomography colonography (CTC) and optical colonoscopy $(\mathrm{OC})$

\begin{tabular}{|c|c|c|c|c|c|c|c|c|c|c|}
\hline & \multicolumn{5}{|c|}{ Female participants } & \multicolumn{5}{|c|}{ Male participants } \\
\hline & \multicolumn{2}{|c|}{ СТC } & \multicolumn{2}{|l|}{ OC } & \multirow[t]{2}{*}{$P$-value } & \multicolumn{2}{|c|}{ СТC } & \multicolumn{2}{|l|}{ OC } & \multirow[t]{2}{*}{$P$-value } \\
\hline & $\mathbf{N}$ & $\%$ & $\mathbf{N}$ & $\%$ & & $\mathbf{N}$ & $\%$ & $\mathbf{N}$ & $\%$ & \\
\hline Refuse any additional screening* & 35 & $2.9 \%$ & 14 & $1.2 \%$ & - & 21 & $2.0 \%$ & 7 & $0.7 \%$ & - \\
\hline Prefer male examiner & 3 & $0.2 \%$ & 12 & $1.0 \%$ & 0.022 & 90 & $8.4 \%$ & 93 & $8.7 \%$ & 0.766 \\
\hline Prefer female examiner & 538 & $44.5 \%$ & 293 & $24.3 \%$ & $<0.001$ & 78 & $7.3 \%$ & 49 & $4.6 \%$ & $<0.001$ \\
\hline No preference & 632 & $52.3 \%$ & 889 & $73.6 \%$ & $<0.001$ & 880 & $82.3 \%$ & 920 & $86.1 \%$ & $<0.001$ \\
\hline
\end{tabular}

Notes: $*$ No $P$-value is shown for participants who planned to refuse additional screening, as these participants did not express a preference for the sex of the examiner. Exact $P$-values from McNemar's test between tests on the proportion within category.

adhere with OC at 10 years", and "Plan to adhere with CTC at 5 years", respectively. ORs greater than one in the column titled "Plan to adhere with $\mathrm{OC}$ at 10 years" indicate factors associated with higher odds of intended adherence to OC at 10 years but not CTC at 5 years. ORs greater than one in the column titled "Plan to adhere with CTC at 5 years" indicate the reverse - factors associated with higher odds of intended adherence with CTC but not OC.

Plans to adhere to OC screening guidelines in the future, as opposed to willingness to adhere to guidelines for both $\mathrm{OC}$ and CTC was associated with being female $(\mathrm{OR}=1.85 ; 95 \%$ CI: 1.44-2.38), worse than expected discomfort with the BP $(\mathrm{OR}=1.76$; 95\% CI: 1.32-2.33), worse than expected physical discomfort from CTC (OR=3.35; 95\% CI: 2.58-4.33), embarrassment during the CTC procedure ( $\mathrm{OR}=1.57 ; 95 \%$ CI: $1.22-2.01)$, and not having a biopsy ( $\mathrm{OR}=1.67 ; 95 \%$ CI: 1.29-2.18).

Plans to adhere to CTC screening guidelines in the future, as opposed to willingness to adhere to guidelines for both $\mathrm{OC}$ and CTC was associated with worse than expected physical discomfort from OC (OR=10.56: 95\% CI: 2.94-37.92), worse than expected embarrassment from $\mathrm{OC}(\mathrm{OR}=7.56$; $95 \%$ CI: 1.28-44.45), and time away from usual activities. Each hour spent away from usual activities was associated with a $10 \%$ increase in the odds of a patient being willing to be screened with CTC as opposed to both OC and CTC within the recommended interval (95\% CI: 1.02-1.18).

\section{Discussion}

Although NCTCT participants expressed a preference for CTC relative to OC, their willingness to undergo repeat CTC within the recommended screening interval appeared to be limited by unanticipated exam discomfort and embarrassment and the shorter interval time between screenings currently recommended for CTC as opposed to OC.

Our finding, that a majority of NCTCT participants expressed a preference for CTC over OC, is consistent with previous reports. ${ }^{25-27}$ This preference may be due to the shorter overall examination time with CTC as opposed to $\mathrm{OC}$ as well as patient preference for noninvasive screening methods. ${ }^{28}$ The shorter examination time was important to NCTCT participants. In our multivariate model, the odds of patients planning to return for a CTC screen, as opposed to both CTC and OC, within the recommended interval increased proportionally with the time spent away from usual activities for the two NCTCT screening tests. In addition, the expressed preference for CTC in the NCTCT may reflect a volunteer effect, with patients who chose to participate in the NCTCT having already revealed a willingness to undergo CTC. This volunteer effect may help to explain the apparent inconsistency between the expressed preference for CTC and patient willingness to return within the current recommended screening interval.

In our study, $79.3 \%$ of participants were willing to return for CTC within the recommended 5 years, whereas $96.6 \%$ were willing to return for $\mathrm{OC}$ within the recommended 10 years. In contrast, de Wijkerslooth et $\mathrm{al}^{21}$ reported that 93\% of CTC participants and $96 \%$ of OC participants indicated that they would probably or definitely participate in the next screening round. The difference between the two studies in the proportion of CTC participants willing to return is probably attributable to several factors. Most importantly, the NCTCT used a cathartic BP whereas de Wijkerslooth et $\mathrm{a}^{21}$ reported results from noncathartic CTC. In addition, the NCTCT questionnaire specifically stated the time to the next screening test ( 5 years for CTC as opposed to 10 years for $\mathrm{OC}$ ), it is unclear if this was the case in the questionnaire administered by Wijkerslooth et al. ${ }^{21}$ Finally, NCTCT participants were willing to undergo both screening modalities, whereas participants in the Dutch study were randomly assigned to be offered either CTC or OC. The proportion of patients who accepted CTC (34\%) was substantially higher than the proportion who accepted OC $(22 \%)$. This difference in participation rates across the arms might have influenced the expressed intention to participate in future screening. 


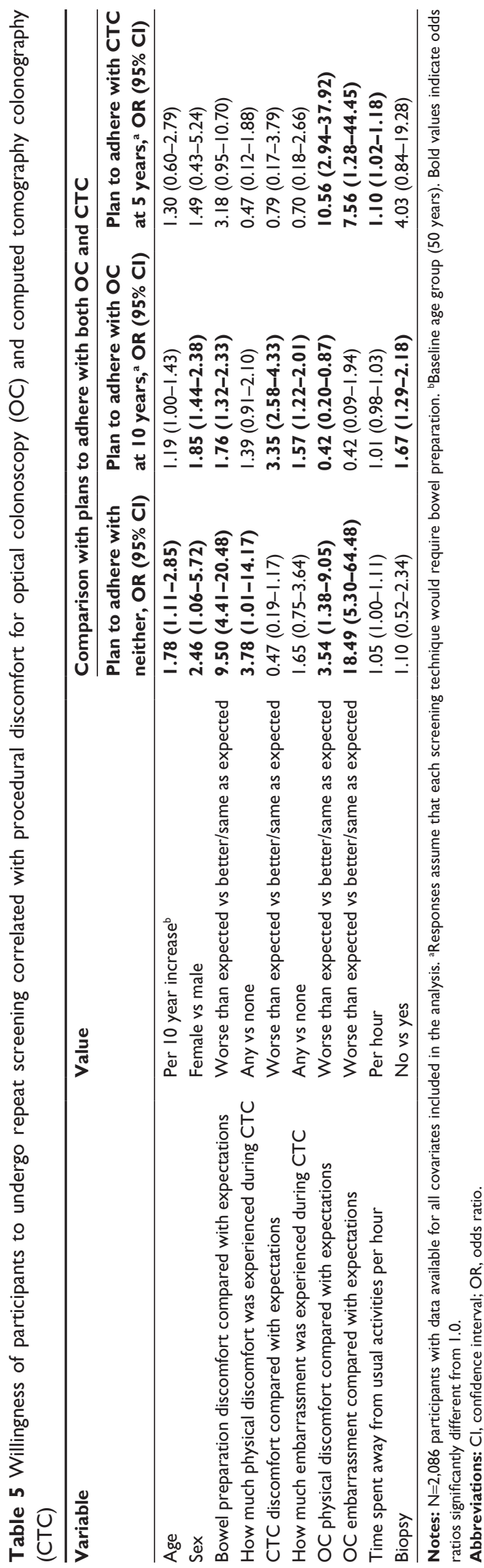

Much of the unwillingness to return for CTC in the NCTCT appears to be due to discomfort and embarrassment associated with CTC. Worse than expected physical discomfort with CTC and embarrassment during the CTC procedure were strongly associated with increased odds of patient willingness to adhere with only OC. This finding is of importance because a substantial proportion of participants reported that their discomfort was worse than expected with CTC (32.9\%), and a sizable number reported that embarrassment was worse than expected with CTC (8.5\%). The contrast between expectations and actual experience may be addressable with enhanced patient education. Improving and tailoring preprocedural counseling to better explain the CTC experience and potential side effects may be effective in reducing the proportion of CTC participants who experience a higher level of discomfort and embarrassment than anticipated. ${ }^{21}$ In addition, new techniques, such as partially deflating the colon, may relieve discomfort associated with CTC screening. ${ }^{29}$ Interestingly, worse than expected discomfort and embarrassment with OC were associated with planning to adhere with only CTC and planning to adhere with neither screening regimen in the future. The proportion of participants reporting "worse than expected discomfort" with OC (5.0\%) and "worse than expected embarrassment" with OC (1.6\%), however, was substantially smaller.

The higher prevalence of patient-reported physical discomfort and embarrassment with CTC than with OC (discomfort in $81.3 \%$ of CTC and $27.8 \%$ of OC) may be attributable to the routine use of conscious sedation for OC. Conscious sedation may dim memory of pain, discomfort, or embarrassment associated with OC. ${ }^{30}$ Lower levels of conscious sedation have been associated with patient reports that OC was more painful than CTC. ${ }^{31}$ Patients at higher risk of colorectal cancer may also be less likely to report pain and discomfort associated with a screening study. ${ }^{25,26}$

Short screening intervals (Table 2) were associated with an unwillingness to return for rescreening no matter the modality, as was the BP. NCTCT participants reported that they were more likely to return for OC than for CTC at a 10 -year screening interval. This is consistent with reports by Gluecker et al. ${ }^{20}$ This may be due to the potential disadvantage of CTC, which requires patients to return for polyp removal. Patients who were previously diagnosed with colorectal polyps have been reported to express a preference for OC over CTC; ${ }^{26}$ however, this not did not appear to be the case in NCTCT. In fact, patients who did not have a biopsy were more likely to be willing to return for only OC, as opposed to both modalities, than were those who had a 
biopsy, probably because few of these biopsies resulted in a cancer diagnosis. ${ }^{22}$

BP also significantly influenced participant willingness to return for screening. Most participants reported that they would be willing to return for both CTC and OC screening within the recommended intervals, if BP were unnecessary. While the elimination of BP is not an option for OC at the moment, several studies have pursued CTC without cathartic preparation with encouraging success. ${ }^{16-19,32}$ Although participants invited for noncathartic CTC have been more likely to accept screening, than those invited for $\mathrm{OC}$, the rate of advanced neoplasia detection was lower for noncathartic CTC than for OC. ${ }^{31}$

Our study had several potential limitations. First, our study population consisted of a highly motivated group of participants who were already committed to obtaining a screening colonoscopy at the time of study recruitment. These motivated participants may report higher levels of intended future adherence than the general screening population. Second, this was not a Phase III clinical trial in which participants were randomly assigned to CTC or OC. Each participant was screened using both screening modalities. The study design mandated that CTC be performed prior to OC. This nonrandomized sequence may have influenced patient perceptions, favoring CTC in some way. Third, the sedation used during OC may have obscured participant recall of the discomfort level associated with OC. Even if this were the case, however, discomfort experienced but forgotten is unlikely to influence the patient's determination of whether or not to return for repeat screening.

\section{Conclusion}

CTC holds promise as a colon screening procedure, but to ensure that patients are willing to return for routine screening, patient education and consent need to fully explain the potential discomfort and embarrassment associated with the procedure. A better patient understanding of CTC may improve intended adherence with future screening procedures. In addition, the development of new methods that do not require BP should improve patient intent to return for repeat screening within the recommended screening intervals. Finally, our findings suggest the need for better understanding of patient preferences for the examiner's sex, particularly among women undergoing CTC.

\section{Acknowledgments}

The authors thank the Screening Center investigators and staff of ACRIN 6664: the National CT Colonography Trial
(NCTCT). Most importantly, we acknowledge the study participants, whose contributions made this study possible. We also acknowledge Maryann Duggan, Cheryl Souza, and Nancy Ciminelli for their diligent work in data collection for this study. This study was supported by the National Cancer Institute grant CA 79778 under a cooperative agreement with the Cancer Imaging Program.

\section{Disclosure}

Dr Gareen reports grants from the National Cancer Institute, during the conduct of the study. Dr Siewert and Mr Herman have nothing to disclose. Dr Vanness reports grants from American College of Radiology Imaging Network during the conduct of the study. Dr Gatsonis reports grants from the National Cancer Institute, during the conduct of the study; personal fees and other from Wilex AG, personal fees and other from Endocyte Inc, other from Frontier Science Research Foundation, personal fees from Genentech, personal fees from Biomimetics, outside the submitted work. Dr Johnson reports personal fees from GE Medical, outside the submitted work; in addition, Dr Johnson has a patent CT Colonography issued.

\section{References}

1. Siegel R, Naishadham D, Jemal A. Cancer statistics, 2012. CA Cancer J Clin. 2012;62(1):10-29.

2. Smith RA, Cokkinides V, Eyre HJ. Cancer screening in the United States, 2007: a review of current guidelines, practices and prospects. CA Cancer J Clin. 2008;58:161-179

3. US Preventive Services Task Force (USPSTF). Screening for colorectal cancer: U.S. Preventive Services Task Force recommendation statement. Ann Intern Med. 2008;149(9):627-637.

4. Schoen RE, Pinsky PF, Weissfeld JL, et al; PLCO Project Team. Colorectal cancer incidence and mortality with screening flexible sigmoidoscopy. N Engl J Med. 2012;366(25):2345-2357.

5. Hardcastle JD, Chamberlain JO, Robinson MH, et al. Randomized controlled trial of fecal-occult-blood screening for colorectal cancer. Lancet. 1996;348:1472-1477.

6. Mandel JS, Bond JH, Church TR, et al. Reducing mortality from colorectal cancer by screening for fecal occult blood. N Engl J Med. 1993; 328:1365

7. Kronborg O, Fenger C, Olsen J, Jorgensen OD, Sondergaard O. Randomised study of screening for colorectal cancer with fecal-occult-blood test. Lancet. 1996;348:1467-1471.

8. Pignone M, Saha S, Hoerger T, Mandelblatt J. Cost-effectiveness analyses of colorectal cancer screening: a systematic review for the U.S Preventive Services Task Force. Ann Intern Med. 2002;137:96-104.

9. Zauber AG, Landsdorp-Vogelaar II, Knudsen AB, Wilschut J, van Ballegooijen M, Kuntz KM. Age to Begin, Age to End, and Timing of Screening Intervals: Decision Analysis of Colorectal Cancer Screening for the United States Preventive Services Task Force from the Cancer Intervention and Surveillance Modeling Network (CISNET). Evidence Synthesis No. 65, Part 2. AHRQ Publication No. 08-05124EF-2. Rockville, MD: Agency for Healthcare Research and Quality; 2008.

10. American Cancer Society. Colorectal Cancer Facts \& Figures 2011-2013. Atlanta, GA: American Cancer Society; 2011. 
11. Zarychanski R, Chen Y, Bernstein CN, Hébert PC. Frequency of colorectal cancer screening and the impact of family physicians on screening behavior. CMAJ. 2007;177:593-597.

12. Seeff LC, Nadel MR, Klabunde CN, et al. Patterns and predictors of colorectal cancer test use in the adult U.S. population. Cancer. 2004;100: 2093-2103.

13. Levin B, Lieberman DA, McFarland, et al. Screening and surveillance for the early detection of colorectal cancer and adenomatous polyps, 2008: a joint guideline from the American Cancer Society, the US Multi-Society Task Force on Colorectal Cancer, and the American College of Radiology. CA Cancer J Clin. 2008;58:130-160.

14. Agostoni M, Fanti L, Gemma M, Pasculli N, Beretta L, Testoni PA. Adverse events during monitored anesthesia care for GI endoscopy: an 8-year experience. Gastrointest Endosc. 2011;74(2):266-275.

15. Marshall DA, Johnson FR, Phillips KA, Marshall JK, Thabane L, Kulin NA. Measuring patient preferences for colorectal cancer screening using a choice-format survey. Value Health. 2007;10(5):415-430.

16. Callstrom MR, Johnson CD, Fletcher JG, et al. CT colonography without cathartic preparation: feasibility study. Radiology. 2001; 219(3):693-698.

17. Zalis ME, Hahn PF. Digital subtraction bowel cleansing in CT colonography. AJR Am J Roentgenol. 2002;176:646-648.

18. Iannacone R, Laghi A, Catalano C, et al. Computed tomographic colonography without cathartic preparation for the detection of colorectal polyps. Gastroenterology. 2004;127:1300-1311.

19. Zalis ME, Blake MA, Cai W, et al. Diagnostic accuracy of laxative-free computed tomographic colonography for detection of adenomatous polyps in asymptomatic adults: a prospective evaluation. Ann Intern Med. 2012;156(10):692-702.

20. Gluecker TM, Johnson CD, Harmsen WS, et al. Colorectal cancer screening with CT colonography, colonoscopy, and double-contrast barium enema examination: prospective assessment of patient perceptions and preferences. Radiology. 2003;277:378-384.

21. de Wijkerslooth TR, de Haan MC, Stoop EM, et al. Burden of colonoscopy compared to non-cathartic CT-colonography in a colorectal cancer screening programme: randomised controlled trial. Gut. 2012; 61(11):1552-1559.
22. Johnson CD, Chen MH, Toledano AY, et al. Accuracy of CT colonography for detection of large adenomas and cancers. N Engl J Med. 2008; 359:1207-1217.

23. Johnson CD, Chen MH, Toledano AY, et al. ACRIN 6664: National CT Colonography Trial [Protocol]. American College of Radiology, August 4, 2010. Available from: http://www.acrin.org/Portals/0/ Protocols/6664/ACRIN_6664_AdminUpdate_080410_master.pdf. Accessed May 19, 2015.

24. http://www.acrin.org/Portals/0/Protocols/6664/forms/6664participant_ ques.pdf. Accessed May 19, 2015.

25. Svenson MH, Svenson E, Larson A, Hellstroem M. Patient acceptance of CT colonography and conventional colonoscopy: prospective comparative study in patients with or suspected of having colorectal disease. Radiology. 2002;222:337-345.

26. Van Gelder RE, Birnie E, Florie J, et al. CT colonography and colonoscopy: assessment of patient preference in a 5-week follow-up study. Radiology. 2004;233:328-337.

27. Lin OS, Kozarek RA, Gluck M, et al. Preference for colonoscopy versus computerized tomographic colonography: a systematic review and meta-analysis of observational studies. J Gen Intern Med. 2012;27(10): 1349-1360.

28. Ling BS, Moskowitz MA, Wachs D, Pearson B, Schroy PC. Attitudes toward colorectal cancer screening tests. J Gen Intern Med. 2001; $16: 822-830$

29. Dachman AH. Advice for optimizing colonic distention and minimizing risk of perforation during CT colonography. Radiology. 2006;239(2): 317-321.

30. Ristvedt SL, McFarland EG, Weinstock LB, Thyssen EP. Patient preference for CT colonography, conventional colonoscopy, and bowel preparation. Am J Gastroenterol. 2003;98:578-585.

31. Forbes GM, Mendelson RM. Patient acceptance of virtual colonoscopy. Endoscopy. 2000;32:272.

32. Stoop EM, de Haan MC, de Wijkerslooth TR, et al. Participation and yield of colonoscopy versus non-cathartic CT colonography in population-based screening for colorectal cancer: a randomized controlled trial. Lancet Oncol. 2012;13(1):55-64. 


\section{Supplementary material}

Permission to conduct the study was obtained from the institutional review boards of: Brown University, Providence, RI; Mayo Clinic, Scottsdale, Scottsdale, AZ; Scottsdale Medical Imaging, Ltd., Scottsdale, AZ; UCLA School of Medicine, Los Angeles, CA; UCSD Medical Center, San Diego, CA; San Francisco VA Medical Center (SFVAMC), San Francisco, $\mathrm{CA}$; Radiology Imaging Associates, Englewood, CO;
Yale University, New Haven, CT; University of Chicago Hospital, Chicago, IL; Clinical Radiologists, S.C. Memorial Medical Center; Springfield, IL; Johns Hopkins, Baltimore, MD; Beth Israel Deaconess Medical Center, Boston, MA; Mayo Clinic, Rochester, MN; Washington University Medical School, St Louis, MO; MD Anderson, Houston, TX; and Medical College of Virginia Hospitals, Richmond, VA.

\section{Publish your work in this journal}

Patient Preference and Adherence is an international, peer-reviewed, open access journal that focuses on the growing importance of patient preference and adherence throughout the therapeutic continuum. Patient satisfaction, acceptability, quality of life, compliance, persistence and their role in developing new therapeutic modalities and compounds to optimize clinical outcomes for existing disease states are major areas of interest for the journal. This journal has been accepted for indexing on PubMed Central. The manuscript management system is completely online and includes a very quick and fair peer-review system, which is all easy to use. Visit http://www. dovepress.com/testimonials.php to read real quotes from published authors. 